University of Louisville

ThinkIR: The University of Louisville's Institutional Repository

\title{
Sinusoidal endothelial cell-derived extracellular matrix regulates basal and stimulated macrophage activation.
}

Lauren G. Poole

University of Louisville

Follow this and additional works at: https://ir.library.louisville.edu/honors

Part of the Biology Commons, and the Pharmacology, Toxicology and Environmental Health Commons

\section{Recommended Citation}

Poole, Lauren G., "Sinusoidal endothelial cell-derived extracellular matrix regulates basal and stimulated macrophage activation." (2013). College of Arts \& Sciences Senior Honors Theses. Paper 21.

http://doi.org/10.18297/honors/21

This Senior Honors Thesis is brought to you for free and open access by the College of Arts \& Sciences at ThinkIR: The University of Louisville's Institutional Repository. It has been accepted for inclusion in College of Arts \& Sciences Senior Honors Theses by an authorized administrator of ThinkIR: The University of Louisville's Institutional Repository. This title appears here courtesy of the author, who has retained all other copyrights. For more information, please contact thinkir@louisville.edu. 


\section{SINUSOIDAL ENDOTHELIAL CELL-DERIVED EXTRACELLULAR MATRIX}

REGULATES BASAL AND STIMULATED MACROPHAGE ACTIVATION

\section{By}

Lauren G. Poole

Submitted in partial fulfillment of the requirements

for Graduation (summa or magna) cum laude and

For Graduation with Honors from the Department of Biology

University of Louisville

May 2013 


\section{Acknowledgements}

I would like to thank my mentor, Dr. Gavin Arteel for his constant guidance and encouragement. I am grateful for the opportunities he has provided for me as an undergraduate researcher and for the skills I have learned as his student. I would also like to thank my coworkers for their assistance with this project, and my committee members, Dr. Cynthia Corbitt and Dr. Michael Perlin for their valuable suggestions.

I would also like to acknowledge the University of Louisville Summer Research Opportunities Program for funding this work. 


\begin{abstract}
SINUSOIDAL ENDOTHELIAL CELL-DERIVED EXTRACELLULAR MATRIX REGULATES BASAL AND STIMULATED MACROPHAGE ACTIVATION

Lauren G. Poole
\end{abstract}

$03 / 29 / 2013$

Background. Fatty liver disease, be it from alcohol (ALD), obesity (NAFLD) or other sources (e.g. viral infection), share similar mechanisms of disease progression. One potential mechanism is that altered sinusoidal endothelial cell (SEC) extracellular matrix (ECM) favors a more inflammatory phenotype in resident macrophages (i.e., Kupffer cells). Here, the hypothesis was tested that SEC-derived matrices directly affect the inflammatory response of macrophages. Methods. Transformed sinusoidal endothelial cells (TSECs) were cultured for 24 or 72 hours. Culture plates were then washed with a solution that selectively removed the cells, but preserved the ECM. Cultured macrophages (RAW 264.7 cells) were then seeded on the matrix and cultured for 24 hours; the cells were then stimulated with LPS for $0,3,6,12$, or 24 hours $(100 \mathrm{ng} / \mathrm{mL}) \pm$ CycloRGDfV. Real time RT-PCR was used to measure mRNA expression of proinflammatory mediators (IL-6, IL-1 $\beta$, TNF- $\alpha$, and INOS) and anti-inflammatory mediators (IL-10 and TGF- $\beta$ ). Results. LPS stimulated the production of all mediators by macrophages; when plated on ECM from TSECS, this response was attenuated for IL-6 and IL-1 $\beta$. The presence of TSEC-derived ECM increased expression of TGF- $\beta$. The addition of the small peptide antagonist CycloRGDfV produced varying effects on the expression of inflammatory mediators in the presence or absence of TSEC-derived ECM. Conclusions. These data serve as first proof-of-concept that macrophage 
activation can be modulated by ECM produced by TSECs and identifies a new interaction between these cells that may contribute to inflammatory liver disease. 


\section{Introduction}

Liver disease currently affects more than 30 million people in the United States (1). Liver disease can occur in the presence or absence of alcohol consumption, but in both cases includes a spectrum of diseases, from simple steatosis, or fatty accumulation on the liver, to active inflammation, to advanced fibrosis and cirrhosis (2). One potential mechanism for the induction of liver disease is the "two-hit" model, in which underlying factors (e.g. alcohol consumption or environmental factors) sensitizes the liver to a second injury, such as exposure to lipopolysaccharide (LPS) (3).

It is proposed that subtle changes in the hepatic extracellular matrix, either in protein composition or amount, caused by a first hit, such as alcohol or arsenic, can sensitize the liver to a second insult. Previous studies have shown that extracellular matrix remodeling, specifically accumulation of the ECM protein fibrin, plays a critical role in the early development of experimental inflammatory liver injury. In one study, male C57BL/6J, B6.129-Tnfrsf1 $\mathrm{a}^{\mathrm{tm} 1 \mathrm{Mak} / J}\left(\mathrm{TNFR}^{-1^{-/-}}\right)$mice (6 weeks) received ethanol in their diet $(6 \mathrm{~g} / \mathrm{kg})$ for three days (4). Twenty-four hours after the last ethanol administration, mice were injected with LPS, which served as a second hit. Mice were also given Hirudin, U0126, or PAI-1 inactivating antibody to block fibrin accumulation. The results of this study, as shown through histology and plasma enzyme levels, indicated that ethanol consumption enhanced injury caused by LPS. Enhanced injury caused by alcohol correlated to increased accumulation of fibrin ECM by alcohol. Additionally, liver injury and inflammation were dramatically blunted by blocking fibrin deposition. These results indicate that ethanol-induced liver injury is mediated, at least in part, by the 
accumulation of fibrin ECM in the sinusoidal space However, the mechanisms underlying the sensitization of the liver to a second hit are incompletely understood.

Additionally, a study was conducted to test the hypothesis that low levels of arsenic sensitize the liver to injury in a mouse model of non-alcoholic fatty liver disease (5). Male C57BI/6J mice were exposed to low fat diet (LFD; $13 \%$ calories as fat) or high fat $\operatorname{diet}$ (HFD; $42 \%$ calories as fat) and tap water or arsenic in their drinking water (4.9 ppm as sodium arsenite) for ten weeks. Plasma enzyme levels and histology were used to determine the level of liver damage. High fat diet significantly increased body weight gain in mice compared with low-fat controls. HFD significantly increased liver to body weight ratios and caused steatohepatitis. Arsenic exposure had no effect on indices of liver damage in LFD-fed animals however it significantly increased the liver damage caused by HFD. The liver damage enhanced by arsenic correlated with increased inflammation and fibrin extracellular matrix (ECM) accumulation. Taken together, the results of these preliminary studies suggest that hepatotoxins, including arsenic and alcohol, may cause an altered ECM profile in the area of the sinusoids and that this altered ECM may be pro-inflammatory. Therefore, the current study was designed to further explore the mechanisms underlying pro-inflammatory effects of altered ECM in the liver.

One mechanism by which ECM components may contribute to inflammation is through interaction with cell surface adhesion receptors known as integrins (6). For example, fibrin ECM interacts with the integrin $\alpha_{v} \beta_{3}$, a receptor known to contribute to inflammation, tumor angiogenesis, and metastasis $(7,8)$. Integrin $\alpha_{v} \beta_{3}$ binds fibrin ECM through interaction with the RGD (arginine-glycene-aspartate) sequence on the ECM 
(7). When this interaction is blocked using the small peptide antagonist CycloRGDfV, LPS-induced liver injury caused by acute alcohol exposure is blunted with no effect on fibrin accumulation (6). These data suggest that liver injury and inflammation may be due, at least in part, to interaction between fibrin ECM and the integrin $\alpha_{v} \beta_{3}$.

This study focused on the role of two non-parenchymal hepatic cell types that may mediate the effects of ECM on inflammation: sinusoidal endothelial cells and Kupffer cells. Sinusoidal endothelial cells (SECs) line the hepatic sinusoids, where ECM often accumulates in the liver. SECs form the barrier that separates the blood vessel from the Space of Disse, but are fenestrated to allow for the filtration of blood. SECs are known to play a critical role in inflammatory liver injury. For example, it was shown that mice lacking ICAM-1 (a receptor that is highly expressed on SECs) are protected from alcohol-induced inflammatory liver injury (9). As mentioned above, fibrin ECM accumulates in the sinusoidal space, and blocking fibrin protects against inflammatory liver injury (4). The accumulation of this ECM, and other ECM components, may be mediated by SECs. However, this hypothesis has never been tested.

Kupffer cells are also well-known to play a key role in liver injury and inflammation. Kupffer cells are the resident macrophages of the liver. They participate in the innate immune response through the phagocytosis of foreign substances and through the release of inflammatory cytokines. However, in some cases, these cytokines may also damage the surrounding normal tissue. For example, the GI-derived toxin LPS in the bloodstream interacts with Kupffer cells via the CD14/TLR4 receptor complex. Through a series of intracellular signaling cascades, this interaction increases the release of tumor necrosis factor alpha (TNF- $\alpha$ ), which damages the surrounding hepatocytes (10). 
In living systems, SECs and Kupffer cells are constantly interacting and communicating. Therefore, it is important to study the communication between these cells.

It is hypothesized that SEC-derived matrices directly affect the inflammatory response of macrophages. This study will further understanding of the role of SECderived matrices and the effect of these matrices on hepatic macrophage response, which could potentially lead to new therapies. 


\section{Cell culture}

\section{Materials and Methods}

Transformed sinusoidal endothelial cells (TSECs) were a generous gift from Dr. Vijay H. Shah (Mayo Clinic and Foundation, Rochester, MN). TSECs were maintained in DMEM (Sigma) with $5 \%$ fetal bovine serum (FBS), $1 \%$ antibiotic/antimycotic, and endothelial cell grown supplement (Sciencell) and incubated in a humidified $5 \% \mathrm{CO}_{2}$ incubator at $37^{\circ} \mathrm{C}$. Cells were passaged at confluency every $3-4$ days, using a solution of $0.05 \%$ trypsin with EDTA and split by adding $100 \mu \mathrm{L}$ of the trypsinized monolayer to $10 \mathrm{~mL}$ of media. RAW 264.7 cells (ATCC) were maintained in DMEM (Sigma) with 5\% FBS and $1 \%$ antibiotic/antimycotic and incubated in a humidified $5 \% \mathrm{CO}_{2}$ incubator at $37^{\circ} \mathrm{C}$. Cells were passaged every $3-4$ days by gently scraping the side of the culture flask using a cell scraper (no trypsin) and split by adding $1 \mathrm{~mL}$ detached cells to $9 \mathrm{~mL}$ of media.

\section{Treatment of Cells}

TSECs (passage 5-6) were seeded on a 12-well plate (Nunc) at a density of 50,000 cells per well in $1.0 \mathrm{~mL}$ of media. To control for the effect of TSEC-derived ECM, some wells (control wells) were treated in parallel, but devoid of cells. Cells were incubated for 24 or $72 \mathrm{~h}$. After the incubation period, TSECs were washed and treated with a solution containing $0.25 \mathrm{M}$ ammonium hydroxide and $1 \mathrm{mM}$ EDTA to selectively remove the cells and preserve the ECM. After three washes, the extracellular matrix deposited by the TSECs was used as a substratum for culturing RAW 264.7 cells (11, 12). RAW 264.7 cells (passage 4-14) were seeded at a density of 150,000 cells per well in $1.0 \mathrm{~mL}$ of media on the preserved TSEC ECM or in control wells and incubated for 24 hours. Then, cells were exposed to LPS $(100 \mathrm{ng} / \mathrm{mL}) \pm$ CycloRGDfV $(10 \mu \mathrm{M})$ for an 
additional 3, 6, 12, or 24 hour incubation period. Upon termination, supernatant was removed and stabilized with $10 \mu \mathrm{L}$ aprotinin $(1 \mathrm{mg} / \mathrm{mL})$ and stored at $-80{ }^{\circ} \mathrm{C}$ for further analysis. $0.25 \mathrm{~mL}$ RNA STAT was added to each well to lyse the cells and to prepare the lysate for RNA isolation.

\section{RNA Isolation and Quantitative Reverse-Transcriptase Polymerase Chain}

\section{Reaction}

The hepatic mRNA expression of select genes was detected by quantitative reverse-transcriptase polymerase chain reaction (rt-PCR), which is routine for this group $(4,5,13,14)$. PCR primers and probes for TNFa, IL-6, IL-1 $\beta$, iNOS, and $\beta$-actin were designed using Primer 3 (Whitehead Institute for Biomedical Research, Cambridge, MA). Primers and probe for IL-10 were bought from Applied Biosystems as kits (Foster City, CA). All primers were designed to cross introns to ensure that only cDNA and not genomic DNA was amplified. Total RNA was extracted from liver tissue by a guanidinium thiocyanate-based method (RNA STAT 60 Tel-Test, Ambion, Austin, TX). RNA concentrations were determined spectrophotometrically and $1 \mu \mathrm{g}$ of total RNA was reverse transcribed using a kit (Quanta Biosciences, Gaithersburg, MD). PerfeCta qPCR Fast Mix (Quanta Biosciences, Gaithersburg, MD) was used to prepare the PCR reaction mixture. This $2 \mathrm{X}$ mixture is optimized for TaqMan reactions and contains $\mathrm{MgCl}_{2}$, dNTPs, and AccuFast Taq DNA Polymerase. Amplification reactions were carried out using the ABI StepOne Plus machine and software (Quanta Biosciences, Gaithersburg, MD) with initial holding stage $\left(95^{\circ} \mathrm{C}\right.$ for 30 seconds) and 50 cycles of a 2step PCR $\left(95^{\circ} \mathrm{C}\right.$ for 30 seconds, $60^{\circ} \mathrm{C}$ for 20 seconds). Fluorescent intensity of each sample was measured at each cycle to monitor amplification of the target gene. The 
comparative CT method was used to determine fold changes in mRNA expression compared to an endogenous reference gene ( $\beta$-actin). This method determines the amount of target gene, normalized to an endogenous reference and relative to a calibrator $\left(2^{-\Delta \Delta C t}\right)$. 


\section{Results}

TSEC-derived ECM suppresses LPS-induced expression of IL- 6 and IL-1 $\beta$ in RAW

264.7 macrophages. The purpose of this study was to observe the effect of the sinusoidal extracellular matrix on basal and stimulated macrophage activation.

Therefore, the expression of proinflammatory cytokines IL-6, IL-1 $\beta$, and TNF- $\alpha$ was measured over a period of 24 hours by RT-PCR. The TSEC-derived ECM had no effect on basal expression of IL-6, IL-1 $\beta$, and TNF- $\alpha$ in the absence of LPS (Figure 1). LPS alone, however, dramatically increased the expression of all three genes. At 6 hours, the expression of IL- 6 and IL-1 $\beta$ was dramatically decreased by the presence of TSECderived ECM (by $\sim 3$ fold and by $\sim 2.5$ fold, respectively). The expression of TNF- $\alpha$ did not change in the presence of TSEC-derived ECM.

CycloRGDfV produced varying effects on the mRNA expression of IL-6, IL-1 TNF- $\alpha$, iNOS, TGF- $\beta$, and IL-10 in the presence or absence of TSEC-derived ECM. RAW 264.7 cells were grown on either TSEC-derived ECM or in control wells, then exposed to LPS $(100 \mathrm{ng} / \mathrm{mL}) \pm$ CycloRGDfV $(10 \mu \mathrm{M})$ for 6 hours to potentially block the interaction between the ECM and the macrophages. The mRNA expression of IL-6, IL$1 \beta$, TNF- $\alpha$, iNOS, TGF- $\beta$, and IL-10 was determined using real time RT-PCR.

As seen previously (figure1), LPS alone dramatically induced IL-6 expression and the presence of TSEC-derived ECM blunted this effect. The addition of CycloRGDfV further blunted the effect of LPS in both the presence and absence of TSEC-derived ECM (Figure 2, top panel). 
LPS alone also drastically increased the expression of IL-1 $1 \beta$. As seen in Figure 1, the presence of TSEC-derived ECM blunted this effect at the 6 hour time point. Unlike IL-6 expression, the addition of CycloRGDfV increased IL-1 $\beta$ expression in both the presence and absence of TSEC-derived ECM (Figure 2, second panel).

The expression of TNF- $\alpha$ was also increased by the presence of LPS. At the 6 hour time point, TNF- $\alpha$ expression increased in the presence of TSEC-derived ECM, unlike IL- 6 and IL-1 $\beta$ expression. TNF- $\alpha$ expression increased robustly with the addition of CycloRGDfV in the absence of TSEC-derived ECM and increased slightly in the presence of TSEC-derived ECM (Figure 2, third panel).

The expression of iNOS once again increased in the presence of TSEC-derived ECM alone at the 6 hour time point, similar to TNF- $\alpha$ expression. iNOS expression increased robustly with the addition of CycloRGDFV in both the presence and absence of TSEC-derived ECM (Figure 2, bottom panel).

TGF- $\beta$ expression decreased with LPS alone. In the presence of TSEC-derived ECM, TGF- $\beta$ expression increased dramatically. This increase in expression was blunted by the addition of CycloRGDfV (Figure 3, top panel).

IL-10 expression was induced by LPS alone, and the presence of TSEC-derived ECM had no apparent effect on IL-10 expression. The addition of CycloRGDfV decreased IL-10 expression in both the presence and absence of TSEC-derived ECM (Figure 3, bottom panel). 


\section{Discussion}

The aim of this study was to investigate the potential link between the hepatic sinusoidal extracellular matrix and liver injury and inflammation. It has been proposed that hepatotoxins such as alcohol prime macrophages to a second hit, such as LPS. The purpose of this study was to test the hypothesis that SEC-derived matrices directly affect the inflammatory response of macrophages. In order to test this hypothesis, a coculture model was used in which transformed sinusoidal endothelial cells (TSECS) were grown in culture and the matrix produced by these TSECs was isolated. The ECM produced by the TSECs was then used as a substratum for RAW 264.7 cells, which were then exposed to LPS.

RAW 264.7 cells were cultured on a surface containing isolated TSEC ECM or empty surface (control wells). The mRNA expression of several pro- and antiinflammatory mediators was measured in this study. Pro-inflammatory mediators investigated were IL-6, IL-1 $\beta$, TNF- $\alpha$, and iNOS. The IL-6 protein is important in inflammation, B cell maturation, and the development of fever. IL-1 $\beta$ and TNF- $\alpha$ are both critical for cell proliferation, differentiation, and apoptosis (15). iNOS, or inducible nitric oxide synthase, is important in the inflammatory process and defense against pathogens (16). Anti-inflammatory mediators investigated in this study were TGF- $\beta$ (transforming growth factor beta) and IL-10. TGF- $\beta$ is involved in the regulation of proliferation, cell adhesion, and cell migration. IL-10 is another immunoregulatory cytokine (15).

Perhaps the most useful interpretation of the results of this study is the examination of the effect of TSEC-derived ECM on macrophage polarization. Activated 
macrophages are typically polarized into one of two distinct phenotypes as a result of signals from their surrounding environment. These phenotypes are the M1 "classically" activated phenotype and the M2 "alternatively" activated phenotype (17). M1 macrophages are activated by the presence of type 1 cytokines, such as INFy, the recognition of "pathogen-associated molecular patterns" (LPS), or in response to endogenous "danger" signals, like heat shock proteins. M1 macrophages are also involved in macrophage-mediated tissue injury through the release of pro-inflammatory cytokines, including IL-6, IL-1 $1 \beta$, TNF- $\alpha$, and iNOS. In this study, LPS induced the expression of these four M1 pro-inflammatory mediators. In the presence of TSECderived ECM, however, IL-6 and IL-1 $\beta$ expression decreased and TNF- $\alpha$ and iNOS expression increased. With the addition of the small peptide antagonist CycloRGDfV, IL6 expression was dramatically decreased, whereas the expression of M1 mediators IL$1 \beta$, TNF- $\alpha$, and iNOS increased in both the presence and absence of TSEC-derived ECM.

M2, or alternatively activated macrophages, can be subdivided into three classes. M2a macrophages are activated by the presence of cytokines IL-4 and IL-13. M2b macrophages are activated by the presence of immune complexes, IL-1 $1 \beta$, and LPS. M2c macrophages are activated by IL-10, TGF- $\beta$, and glucocorticoids, steroid hormones involved in the immune response (17). M2 macrophages are generally immunosuppressive and are involved in the resolution of inflammation through phagocytosis and tissue repair. They are characterized by the release of antiinflammatory mediators, including TGF- $\beta$ and IL-10. In this study, LPS alone decreased TGF- $\beta$ expression, but increased IL-10 expression. The presence of TSEC-derived 
ECM increased TGF- $\beta$ expression, but had no effect on IL-10 expression at the six hour time point. With the addition of CycloRGDfV, TGF- $\beta$ expression increased in the absence of TSEC-derived ECM, but decreased in the presence of TSEC-derived ECM. IL-10 expression decreased both the presence and absence of TSEC-derived ECM.

The results of this study have several important implications. First, these results indicate that TSEC-derived ECM has a myriad of effects on macrophage polarization. The presence of TSEC-derived ECM shifted the macrophages neither completely to the M1 phenotype, nor the M2 phenotype. For the purposes of this model, therefore, polarization does not appear to shift significantly in one way or the other, and in future studies, macrophage activation should be investigated on a gene-specific basis. Second, the blunting effect produced by the presence of TSEC-derived ECM on the expression of many genes, including IL-6 and IL-1 $1 \beta$, has important implications for invitro work. Future studies should take into consideration that no in-vitro model can completely recapitulate an in-vivo system, especially when cell-ECM communication may play a pivotal role. Finally, the results of this study support the hypothesis that SEC-derived matrices directly affect the inflammatory response of macrophages. While it is not clear if the ECM favors a pro- or anti-inflammatory response, these results suggest that the communication between SECs and Kupffer cells contribute in some way to the inflammatory response, and that this effect may be altered by blocking integrin-ECM interaction.

Previous research from this group has investigated the "two-hit hypothesis," in which in which underlying factors (e.g. alcohol consumption or environmental factors) sensitize the liver to a second inflammatory stimulus, such as exposure to LPS. Indeed, it has 
been shown that both ethanol and sub-hepatotoxic levels of arsenic enhance LPSinduced liver injury. Furthermore, both of these factors are also correlated to changes in ECM composition. The results provided by this study provide valuable information regarding the role of the sinusoidal ECM in regulating macrophage activation in the sensitization of the liver.

In addition to serving as important proof of concept, this study also employed a novel model which will be useful in future studies. While no in vitro model can fully recapitulate the in vivo hepatic environment, the co-culture model used for these studies attempts to recreate one aspect of the hepatic environment, specifically the interaction between sinusoidal endothelial cells and Kupffer cells. In this way, this model can allow for improved in vitro study of important cell-cell interactions compared to other monoculture techniques or models. In the future, further studies would be useful to validate the results produced in this experiment. It would also be useful to characterize the make-up of the ECM produced when TSECs, and potentially are exposed to a stimulus, specifically arsenic.

In conclusion, the results of this study supported the hypothesis that SEC-derived matrices directly affect the inflammatory response of macrophages to a stimulus such as LPS. When RAW 264.7 cells were seeded on a TSEC-derived matrix as opposed to no matrix and exposed to LPS, mRNA expression of the inflammatory mediators IL-6 and IL-1 $\beta$ was severely blunted. These data serve as important proof of concept that SEC-derived ECM may play a role in macrophage activation, providing a potential link between altered sinusoidal ECM composition and hepatic injury. 
Figures

Figure 1

RAW 264.7 cells were cultured on isolated TSEC ECM or control wells for 24 hours, then exposed to LPS (100 ng/mL) for $0,3,6,12$, or 24 hours (see Methods). mRNA expression of IL-6, IL-1 $\beta$, and TNF- $\alpha$ was determined using real time RT-PCR (see Methods).
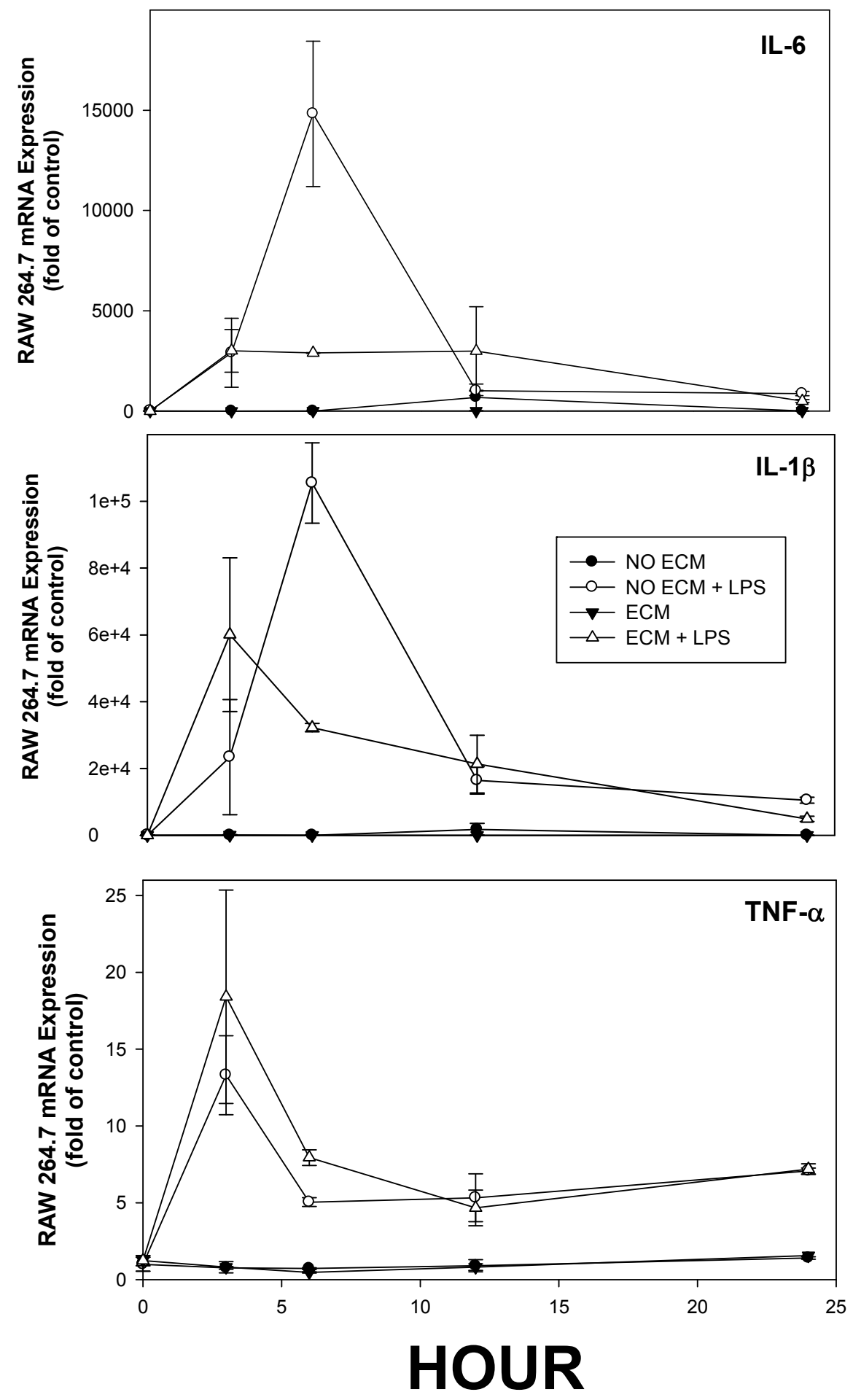


\section{Figure 2}

RAW 264.7 cells were cultured on isolated TSEC ECM or control wells for 24 hours, then exposed to LPS $(100 \mathrm{ng} / \mathrm{mL}) \pm$ CycloRGDfV $(10 \mu \mathrm{M})$ for 6 hours (see Methods). (see Methods). ). mRNA expression of M1 inflammatory mediators IL-6, IL-1 $\beta, T N F-\alpha$, and iNOS was determined using real time RT-PCR (see Methods).

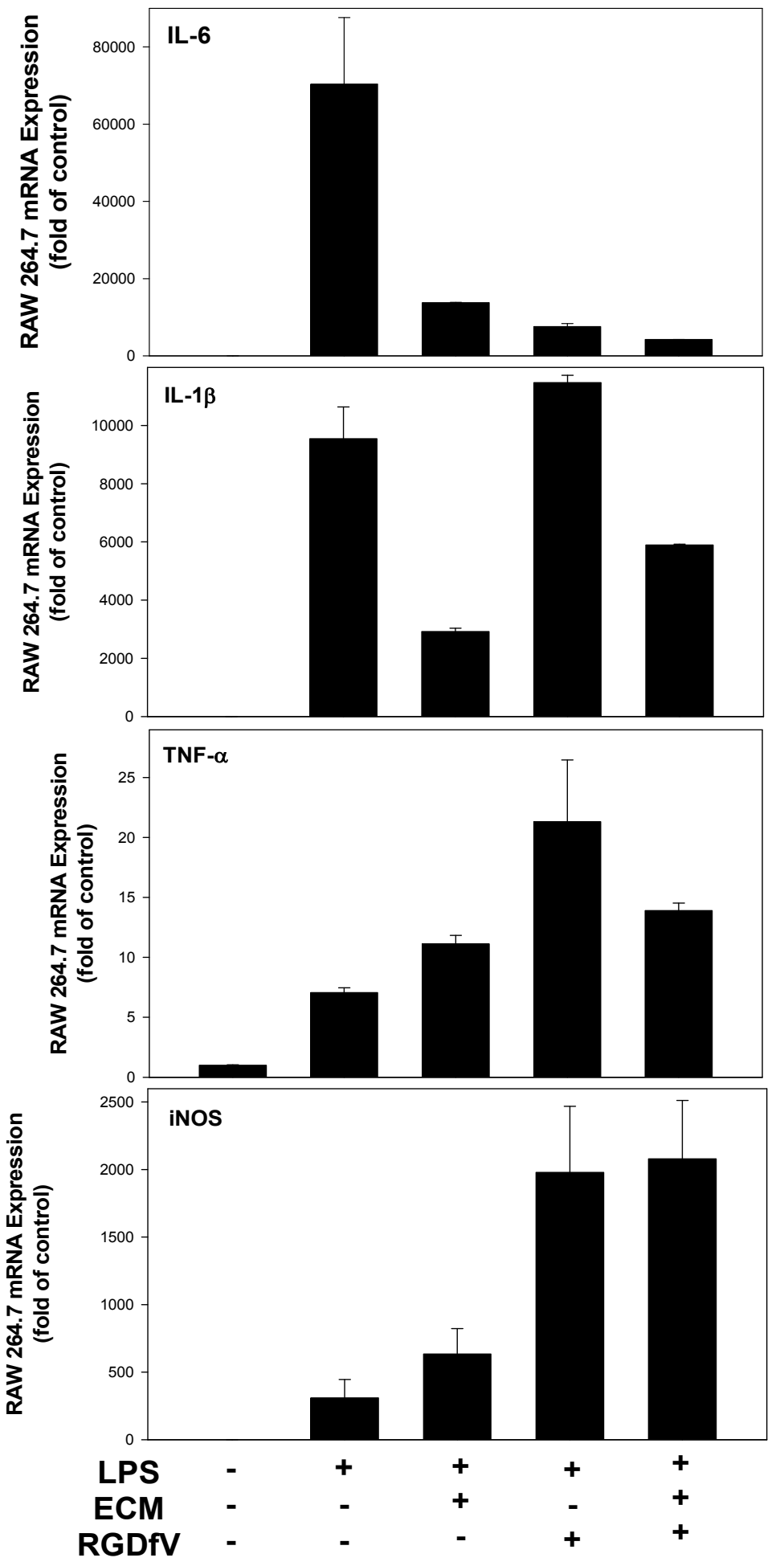


Figure 3

RAW 264.7 cells were cultured on isolated TSEC ECM or control wells for 24 hours, then exposed to LPS $(100 \mathrm{ng} / \mathrm{mL}) \pm$ CycloRGDfV $(10 \mu \mathrm{M})$ for 6 hours (see Methods). (see Methods). ). mRNA expression of M2 anti-inflammatory mediators TGF- $\beta$ and IL-10 was determined using real time RT-PCR (see Methods).
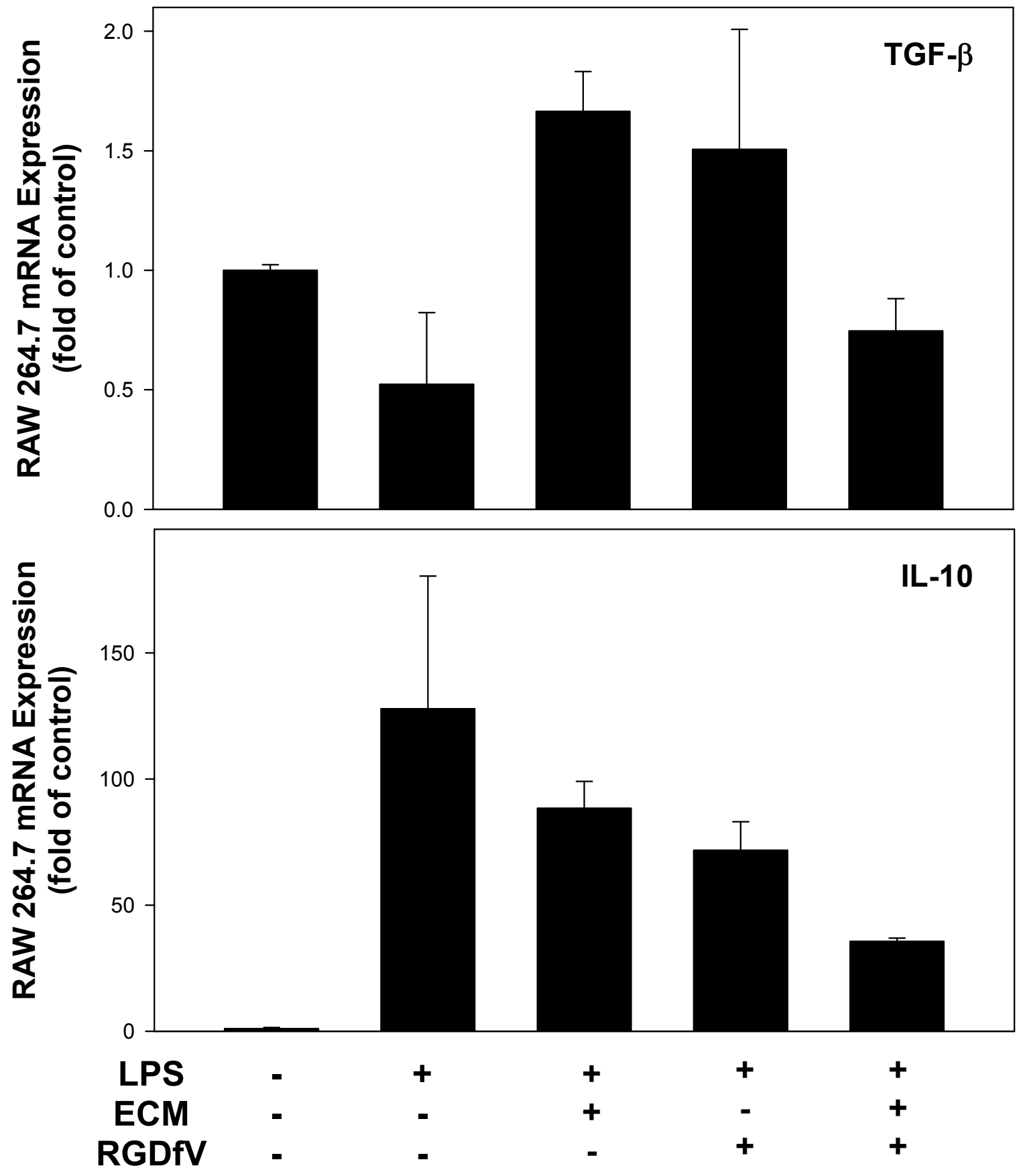


\section{Reference List}

(1) American Liver Foundation. Liver Awareness Month 2010. 2010. http://www.liverfoundation.org/chapters/lam2010

(2) Day CP. Non-alcoholic fatty liver disease: current concepts and management strategies. Clin Med 2006 Jan;6(1):19-25.

(3) Day CP, James OF. Steatohepatitis: a tale of two "hits"? Gastroenterology 1998 Apr;114(4):842-845.

(4) Beier JI, Luyendyk JP, Guo L, von Montfort C, Staunton DE, Arteel GE. Fibrin accumulation plays a critical role in the sensitization to lipopolysaccharide-induced liver injury caused by ethanol in mice. Hepatology 2009 Jan 23;49(5):1545-1553.

(5) Tan M, Schmidt RH, Beier JI, Watson WH, Zhong H, States JC, et al. Chronic subhepatotoxic exposure to arsenic enhances hepatic injury caused by high fat diet in mice. Toxicol Appl Pharmacol 2011 Dec 15;257(3):356-364.

(6) Massey VL, Arteel GE. Acute alcohol-induced liver injury. Frontiers in Physiology 2012;3:1-8.

(7) Cheresh DA, Berliner SA, Vicente V, Ruggeri ZM. Recognition of distinct adhesive sites on fibrinogen by related integrins on platelets and endothelial cells. Cell 1989 Sep 8;58(5):945-953.

(8) Zhou HF, Chan HW, Wickline SA, Lanza GM, Pham CT. Alphavbeta3-targeted nanotherapy suppresses inflammatory arthritis in mice. FASEB J 2009 Sep;23(9):2978-2985.

(9) Kono H, Uesugi T, Froh M, Rusyn I, Bradford BU, Thurman RG. ICAM-1 is involved in the mechanism of alcohol-induced liver injury: studies with knockout mice. Am J Physiol Gastrointest Liver Physiol 2001 Jun;280(6):G1289-G1295.

(10) Arteel GE. Oxidants and antioxidants in alcohol-induced liver disease. Gastroenterology 2003 Mar;124(3):778-790.

(11) Brown LA, Ritzenthaler JD, Guidot DM, Roman J. Alveolar type II cells from ethanolfed rats produce a fibronectin-enriched extracellular matrix that promotes monocyte activation. Alcohol 2007 Aug;41(5):317-324.

(12) Dunsmore SE, Lee YC, Martinez-Williams C, Rannels DE. Synthesis of fibronectin and laminin by type II pulmonary epithelial cells. Am J Physiol 1996 Feb 1;270(2):L215L223. 
(13) Bergheim I, Guo L, Davis MA, Lambert JC, Beier JI, Duveau I, et al. Metformin prevents alcohol-induced liver injury in the mouse: Critical role of plasminogen activator inhibitor-1. Gastroenterology 2006 Jun;130(7):2099-2112.

(14) Guo L, Richardson KS, Tucker LM, Doll MA, Hein DW, Arteel GE. Role of the reninangiotensin system in hepatic ischemia reperfusion injury in rats. Hepatology 2004 Sep;40(3):583-589.

(15) National Center for Biotechnology Information Gene Database. 2013. www.ncbi.nlm.nih.gov/gene/

(16) Lechner M, Lirk P, Rieder J. Inducible nitric oxide synthase (iNOS) in tumor biology: the two sides of the same coin. Semin Cancer Biol 2005 Aug;15(4):277-289.

(17) Laskin DL. Macrophages and Inflammatory Mediators in Chemical Toxicity: A Battle of Forces. Chem Res Toxicol 2009 Aug 17;22(8):1376-1385. 